\title{
Development of a magnetic-resonance-compatible photoplethysmograph amplifier for behavioral and emotional studies
}

\author{
Soon-Cheol Chung, Ji-Hun Kwon, Bongsoo Lee, \\ JeONG-Han Yı, Hyun-Jun KIM, AND GyE-RAE TACK \\ Konkuk University, Chungju, South Korea
}

\begin{abstract}
The purpose of the present study was to develop a magnetic resonance (MR) compatible photoplethysmograph (PPG) system that can measure the raw PPG signal during MR image acquisition. The system consists of an optic sensor that measures the optic signal, an optic cable that transmits a near-infrared optic signal, a signal amplifier, and a filter for noise removal. To minimize interactive noise, only the optic cable and the optic sensor module are located inside the MR room; the signal amplifier and filter are located outside the MR room. An experiment verified that a reliable PPG signal can be obtained without causing a deterioration in the MR image.
\end{abstract}

As compared with other physiological signals and imaging modalities, magnetic resonance (MR) imaging has outstanding spatial resolution but has a shortcoming with respect to temporal resolution. Physiological signals, such as the electroencephalogram (EEG), the electrocardiogram (ECG), the galvanic skin response (GSR), the skin conductance response(SCR), and the photoplethysmograph(PPG), have excellent temporal resolution but poor spatial resolution. To achieve excellent temporal and spatial information simultaneously, a number of studies regarding MR image acquisition with physiological signals are underway.

Recently, a reliable EEG signal measurement technique with MR acquisition has been introduced. During MR image acquisition, the main magnetic field, the gradient magnetic field, and the radio frequency (RF) pulse induce considerable noise on the EEG signal. To minimize induced noise, a carbon electrode is used instead of an $\mathrm{Ag} / \mathrm{AgCl}$ electrode, and the electromagnetic field behind the leads is offset by twisting the leads. A study on induced noise removal by postsignal processing has been carried out (Goldman, Stern, Engel, \& Cohen, 2000), and a real-time noise removal algorithm has been created (Garreffa et al., 2003). A surface coil is used to minimize the effects of the RF pulse (Van Audekerke, Peerters, Verhoye, Sijbers, \& Van der Linden, 2000). Since the MR environment can affect physiological signals, physiological-signal-measuring instruments can distort MR images. To minimize the distortion of MR images, the length of the transmitter is minimized, the measuring instrument is shielded, and the use of metallic materials is minimized (Liu et al., 2000; Obler, Köstler, Weber, Mack, \& Becker, 1999). On the basis of these techniques, it is possible to perform functional magnetic resonance imag- ing (fMRI) and receive an EEG signal simultaneously. Through the use of this technique, it is possible to examine the mechanism by which the central nervous system (CNS) satisfies temporal and spatial resolution at the same time, and studies have been initiated that attempt to clarify the mutual relationship (Laufts et al., 2003; Mirsattari, Lee, Jones, Bihari, \& Ives, 2004; Sammer et al., 2005).

Studies are in progress that examine the mechanism whereby the CNS and the peripheral nervous system (PNS) simultaneously satisfy temporal and spatial resolution, using fMRI and ECG, respectively, but they are still in the beginning stages (Abi-Abdallah et al., 2006; Chung et al., 2006). The mutual relationships between the SCR, the GSR signal, and fMRI have been partially studied, but these investigations have been insufficient, in that they have not clearly examined both central and peripheral nerves (Nagai et al., 2004; Williams et al., 2001). Thus, it is necessary to examine the mechanisms utilized by the CNS and PNS and to study their mutual relationships through the simultaneous measurement of $f M R I$ and physiological signals.

The PPG signal can yield information regarding the autonomic nerves among the peripheral nerves (Demmel, Ridt, \& Olbrich, 2000; Lundström \& Olsson, 2005). To minimize interactive effects with MR, an MR-compatible PPG or pulse oximeter system that uses fiber optic sensor and cable is commercially available (Gehealthcare.com, 2006; Nonin.com, 2006; von Smekal, Seelos, Küper, \& Reiser, 1995). However, these systems are used mainly for patient monitoring and provide only numerical values of blood oxygen saturation and heart rate.

To perform various behavioral and emotional studies in an MR environment, it is necessary to extract sev-

G.-R.Tack, grtack@kku.ac.kr 


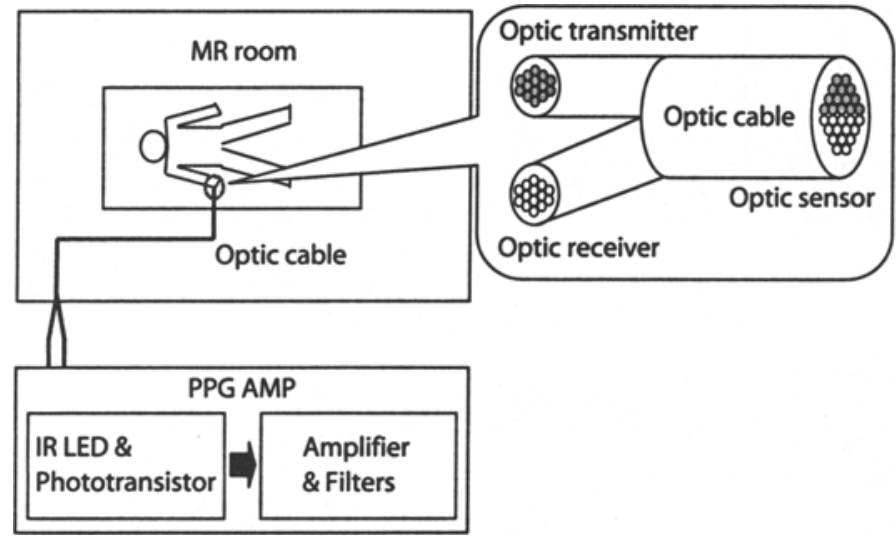

Figure 1. Structure of a magnetic resonance (MR) compatible photoplethysmograph (PPG) system.

eral parameters, such as peak amplitude, peak ratio, time delay between first and second peaks, slope, and so forth from the raw PPG signal (Haba-Rubio et al., 2005; Hofmann, Moscovitch, \& Kim, 2006; Laucevicius, Ryliškytè, Petrulionienè, Kovaitè, \& Misonis, 2004; Millasseau, Kelly, Ritter, \& Chowienczyk, 2003; Roisman, Tsai, \& Chiang, 2004; Woodman et al., 2005). But the development of a PPG system that can extract various parameters for behavioral and emotional study has not been reported. Therefore, in this study, we tried to develop a system that extracts a raw PPG signal with $\mathrm{AMRI}$ in an MR environment. In so doing, we sought to develop a technique that would reliably and simultaneously measure the central and peripheral signals.

\section{METHOD}

\section{MR-Compatible PPG System}

As is shown in Figure 1, this system consists of an optic cable and an optic sensor module, which are located inside the MR room, and a signal amplifier and filter, which are located outside the MR room. The optic cable consists of 36 optic fibers, $1 \mathrm{~mm}$ in diameter and divided into 18 fibers each, which send and receive the optic signal. A bundle that combined each of the 18 optic fibers having been bound, an optic sensor module was built for measuring the PPG signal, using a reflectance method with the sensor in contact with the skin. During measurement, the movement-induced noise is minimized by a band-type housing of contact between the optic sensor and the skin.

The light transmitter, which utilizes an infrared (IR) light-emitting diode (LED), a receiver, and an amplifier, receives reflected light signal from the skin, and the filter is located outside the MR room. The IR LED in the transmitter uses a $650-\mathrm{nm}$ wavelength signal. The phototransistor is a signal receiver that uses a noninverting amplifier. To achieve optimal gain by preventing clipping phenomena, the amplification ratio can be varied up to 1,000 times. A 4th-order low-pass filter is used for highfrequency noise removal, and baseline drift is removed by a high-pass filter. This provides the possibility of measuring a main frequency range of $0.5-20 \mathrm{~Hz}$ for the PPG signal. When the amplifier was located near the operating room, there was considerable electric noise. Thus, the hum was removed by the notch filter.

\section{Test of the MR-Compatible PPG System}

To determine whether the system would operate in a reliable manner, the following experiments were performed. First, the ECG and PPG signals were measured simultaneously to ascertain whether the measured signal was due to movement or other factors. This experiment was performed outside the MR room.

As is shown in Figure 2, to determine whether the optic cable and the optic sensor would induce an MR image to noise, the MR image was measured after the optic cable and optic sensor had been attached to the phantom. To check the region in which the optic sensor and the phantom were in contact, a marker was attached. The image pertaining to the cross section of the marker and the front and rear sections were acquired. T2-weighted images were obtained using a 3.0 Tesla MR system (Magnum 3.0,

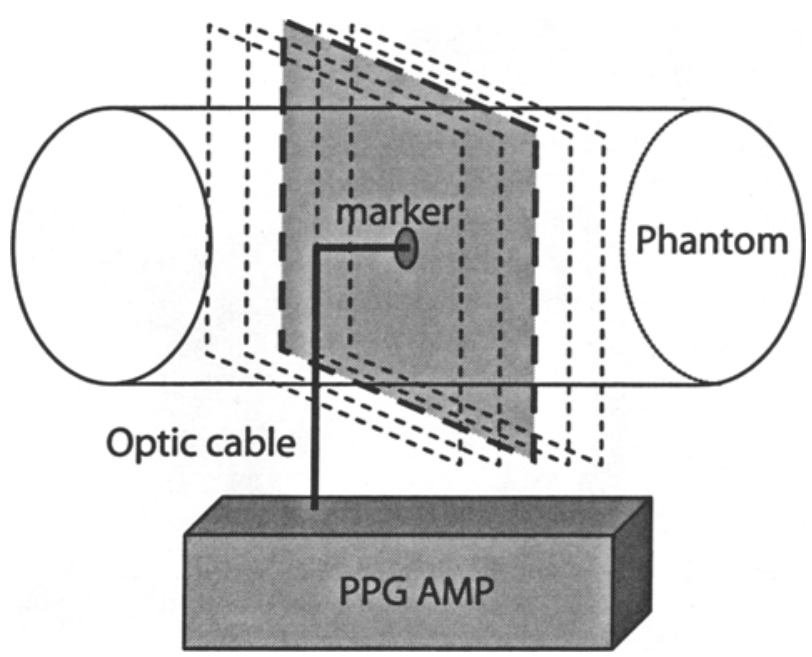

Figure 2. Method for magnetic resonance phantom image acquisition. PPG, photoplethysmograph. 


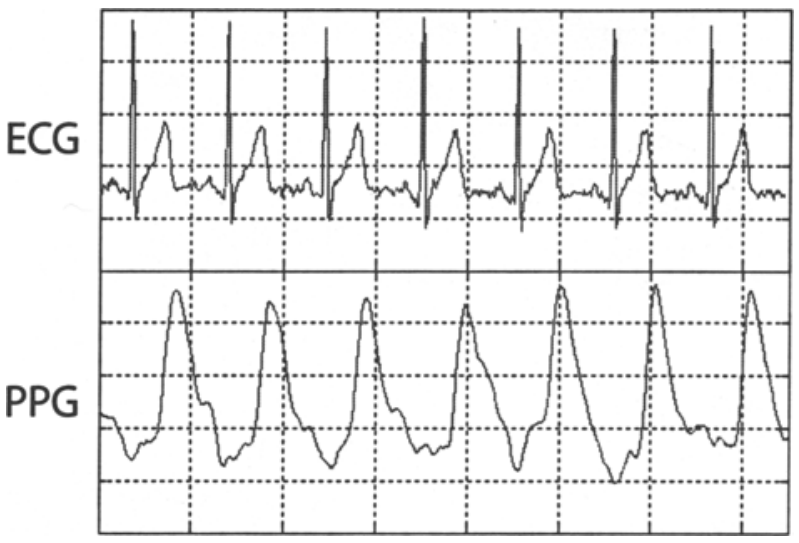

Figure 3. Comparison between electrocardiogram (ECG) and photoplethysmograph (PPG) signals.

Medinus Inc., Korea) with the fast spin echo (FSE) method and the following imaging parameters: TR/TE, $4400 / 96 \mathrm{msec}$; field of view, $240 \mathrm{~mm}$; matrix, $256 \times 256$; slice thickness, $3 \mathrm{~mm}$; and number of slices, 15 .

Finally, the effect of the MR environment on the PPG signal was investigated by performing human subject experiments while measuring the PPG signal and the MR image simultaneously. Images were acquired using the same MR system with the FSE method and the following imaging parameters: TR/TE, $4400 / 96 \mathrm{msec}$; field of view, $240 \mathrm{~mm}$; matrix size, $256 \times 256$; slice thickness, $3 \mathrm{~mm}$; and number of slices, 19 . The PPG signal was also measured while acquiring images with the echo planar imaging (EPI) method, which has been used for fMRI study using the following imaging parameters: TR/TE, $3000 / 96 \mathrm{msec}$; field of view, $240 \mathrm{~mm}$; matrix size, $64 \times$ 64; slice thickness, $3 \mathrm{~mm}$; and number of slices, 9 . The PPG signal, at 300 samples/sec, was measured using a data acquisition board (DAQpad-6015, National Instruments, U.S.). The PPG signal was displayed, saved, and retrieved by using Labview (Version 7.1) and MATLAB (Version 6.5) software.

\section{RESULTS}

As is shown in Figure 3, there was a slight delay between the $R$ point in the ECG signal and the maximum point in the PPG signal; however, a high correlation existed, which indicated that the measured PPG signal was correct. It was verified, as is shown in Figure 4, that neither the optic cable nor the optic sensor caused any deterioration in the MR images.

Two types of MR images were acquired by measuring the PPG signal with human subjects (Figure 5). Figure 6 illustrates the PPG signal measured while MR image acquisition was performed with the EPI method, which showed a slight noise but a reliable signal.

\section{DISCUSSION}

To prevent mutual interference between MR image acquisition and physiological signal measurement, sensors, cables, and amplifiers should not have an effect on MR images. Furthermore, the main magnetic field, gradient magnet field, and RF pulse should not have an effect on physiological signals. Therefore, in the present study, an MR-compatible PPG system was developed using an optic cable and an optic sensor to minimize the mutual interference between the MR image and the raw PPG signal (Gehealthcare.com, 2006; Nonin.com, 2006; von Smekal et al., 1995).

When the physiological-signal-measuring instrument (i.e., the amplifier) was located inside the MR room, metallic material in the instrument had an effect on the MR image. A digital device, such as a microprocessor, induced RF noise, and induced noise had an effect on the MR image. Since the magnetic field and RF pulse of the MR system can cause a noise on the physiological signal measurement system, the instrument requires perfect shielding. To minimize the mutual interference effect in this study, the measuring instrument was located outside the MR room, and only the optic cable and the optic sensor were located inside the MR room.

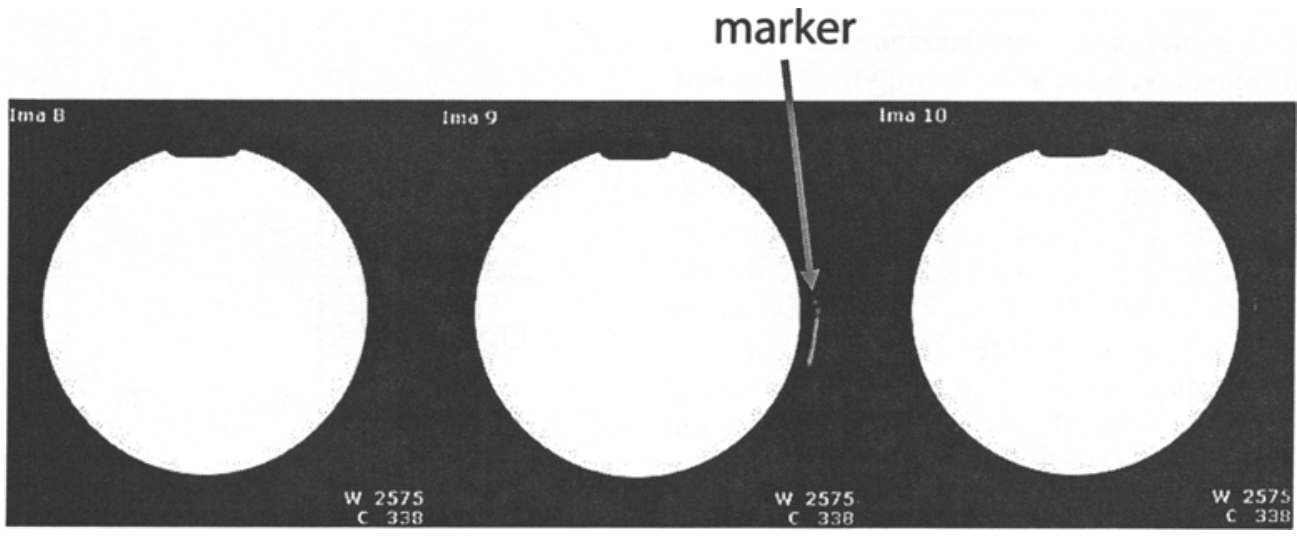

Figure 4. Magnetic resonance phantom images. 


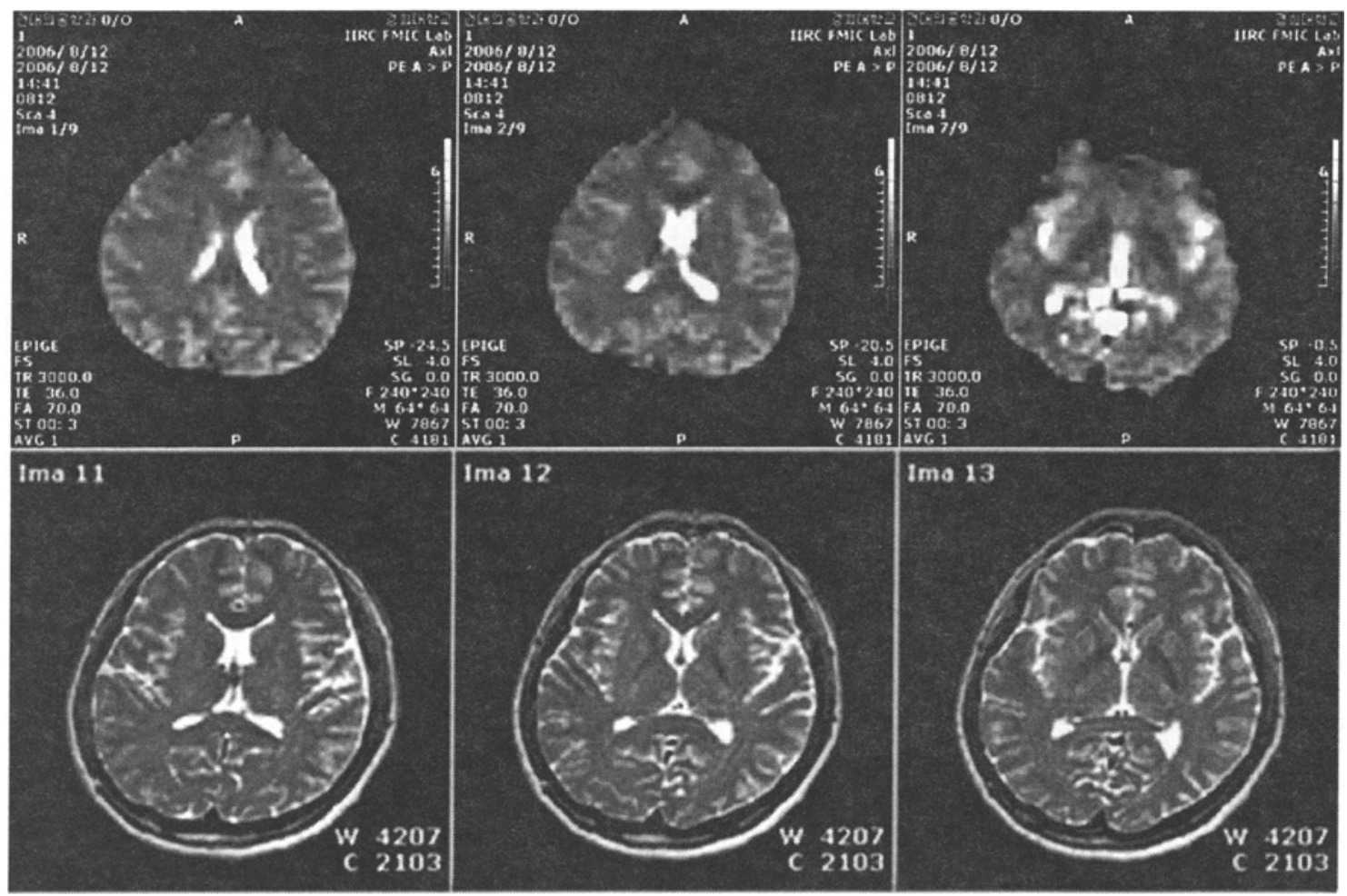

Figure 5. Magnetic resonance images of the subject (upper row, echo planar imaging sequence; lower row, fast spin echo sequence).

In general, the PPG amplifier used a 560- to 940-nm wavelength IR LED, and the wavelength and intensity were selected for the specific application (Ugnell \& Öberg, 1995). This study used a 650-nm IR LED, which inourred the least amount of light loss by considering the distance between a subject in bed inside the MR room and the instrument outside the MR room.

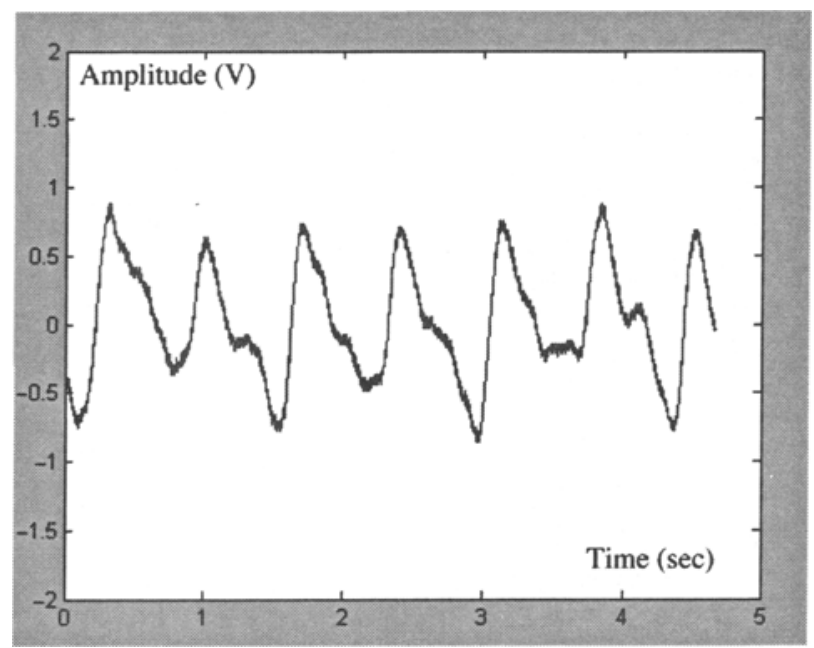

Figure 6. Measured photoplethysmograph signal during magnetic resonance image acquisition with the echo planar imaging method.
When a single optic fiber is used to transmit an optic signal, it is possible to cause a motion artifact on the PPG signal, which is sensitive to movement. The amplitude and quality of the optic signal is increased by increasing the number of optic fibers (Ugnell \& Öberg, 1995). Therefore, an optic cable with a maximum of 36 optic fibers was used in the present study by considering a penetrating hole across the MR room.

The results showed that the optic cable and the optic sensor did not have an effect on the MR image, but the measured PPG signal showed a slight noise. The PPG waveform shape in Figure 6 is different from that in Figure 3. It seems that the PPG waveform acquired during the MR scan is a little bit distorted. There is a possibility that an MR environment, such as a strong magnetic field, may induce physiological changes in the subject and the signal may be slightly distorted, but there are not any published results about this problem. Therefore, it could be concluded that the signal distortion was due to the complex effects of a static magnet field, a gradient magnetic field, and an RF pulse. It will be necessary in future investigations to remove this induced noise.

The findings of this experiment verified that a reliable raw PPG signal can be obtained without causing a deterioration in an MR image. Especially, it is possible to get a reliable PPG signal when MR images are acquired with the EPI method, which has been used for fMRI study. From these raw signals, it is possible to extract various parameters for the purpose of a given study. 
It is expected that this system can be used for diverse behavioral and emotional studies, since it can simultaneously measure the response of the CNS, using fMRI, and that of the PNS, using the PPG.

\section{AUTHOR NOTE}

This research was supported by Grant R11-2002-103 from the Korea Science and Engineering Foundation and Grant 02-PJ3-PG6-EV07-0002 from the Korea Health 21 R\&D Project, Ministry of Health and Welfare, Republic of Korea. Correspondence concerning this article should be addressed to G.-R. Tack, Department of Biomedical Engineering, Research Institute of Biomedical Engineering, College of Biomedical and Health Sciences, Konkuk University, 322 Danwall-dong, Chungju, Chungbuk, 380-701, South Korea (e-mail: grtack@kku.ac.kr).

\section{REFERENCES}

Abi-Abdallah, D., Chauvet, E., Bouchet-Fakri, L., Batalllard, A., BRIGUET, A., \& FoKAPU, O. (2006). Reference signal extraction from corrupted ECG using wavelet decomposition for MRI sequence triggering: Application to small animals. BioMedical Engineering OnLine, 5:11, doi:10.1186/1475-925X-5-11.

Chung, S.-C., YI, J.-H., TACK, G.-R., LeE, B., KwON, J.-H., KIM, S., \& SOHN, J. H. (2006). Development of an MR-compatible ECG amplifier. Key Engineering Materials, 321-323, 1032-1035.

Demmel, R., RidT, F., \& OlBRICH, R. (2000). Autonomic reactivity to mental stressors after single administration of lorazepam in male alcoholics and healthy controls. Alcohol \& Alcoholism, 35, 617-627.

Garreffa, G., Carni, M., Gualniera, G., Ricci, G. B., BozzaO, L., CARLI, D. D., ET AL. (2003). Real-time MR artifacts filtering during continuous EEG/fMRI acquisition. Magnetic Resonance Imaging, 21, 1175-1189.

GEHEALTHCARE.COM (2006). Using the Internet for survey research. Retrieved April 4, 2006, from www.gehealthcare.com accessories $/ 2006 \% 20$ A\&S\%20DI\%20Catalog.pdf.

Goldman, R. I., Stern, J. M., Engel, J., \& COHEN, M. S. (2000). ACquiring simultaneous EEG and functional MRI. Clinical Neurophysiology, 111, 1974-1980.

Haba-Rublo, J., Darbellay, G., Herrmann, F. R., Frey, J. G., FerNANDES, A., VesIN, J. M., ET AL. (2005). Obstructive sleep apnea syndrome: Effect of respiratory events and arousal on pulse wave amplitude measured by photoplethysmography in NREM sleep. Sleep Breath, 9, 73-81.

HofmanN, S. G., Moscovitch, D. A., \& KIM, H. J. (2006). Autonomic correlates of social anxiety and embarrassment in shy and non-shy individuals. International Journal of Psychophysiology, 61, 134-142.

Laucevičcius, A., RYliškyte, L., Petrulionient, Ž., Kovaite, M., \& MisonIs, N. (2004). Photoplethysmographic assessment of the pulse wave: A blunted response to salbutamol in arterial hypertension and coronary artery disease. Seminars in Cardiology, 10, 83-90.

laufts, H., Kleinschmidt, A., Beyerle, A., Eger, E., SalexHaddadi, A., Preibisch, C., \& Krakow, K. (2003). EEG-correlated fMRI of human alpha activity. Neurolmage, 19, 1463-1476.

Liv, J. Z., DaI, T. H., Elster, T. H., SAhGal, V., Brown, R. W., \& YUE, G. H. (2000). Simultaneous measurement of human joint force, surface electromyograms, and functional MRI-measured brain activation. Journal of Neuroscience Methods, 101, 49-57.

LUNDSTRÖM, J. N., \& OLSSON, M. J. (2005). Subthreshold amounts of social odorant affect mood, but not behavior, in heterosexual women when tested by a male, but not a female, experimenter. Biological Psychology, 70, 197-204.

Minlasseau, S. C., Kelly, R. P., Ritter, J. M., \& Chowienczyk, P. J. (2003). The vascular impact of aging and vasoactive drugs: Comparison of two digital volume pulse measurements. American Journal of Hypertension, 16, 467-472.

Mirsattari, S. M., LeE, D. H., Jones, D., Bihari, F., \& IVEs, J. R. (2004). MRI compatible EEG electrode system for routine use in the epilepsy monitoring unit and intensive care unit. Clinical Neurophysiology, 115, 2175-2180.

Nagai, Y., Crttchley, H. D., Featherstone, E., Fenwick, P. B. C., Trmmble, M. R., \& Dolan, R. J. (2004). Brain activity relating to the contingent negative variation: An fMRI investigation. Neurolmage, 21, 1232-1241.

NoNIN.COM (2006). Using the Internet for survey research. Retrieved March 11, 2006, from www.nonin.com/documents $/ 8600 \% 20$ Series\%20Brochure.pdf.

Obler, R., Köstler, H., Weber, B. P., Mack, K. F., \& Becker, H. (1999). Safe electrical stimulation of the cochlear nerve at the promontory during functional magnetic resonance imaging. Magnetic Resonance in Medicine, 42, 371-378.

Roisman, G. I., Tsai, J. L., \& Chiang, K. S. (2004). The emotional integration of childhood experience: Physiological, facial expressive, and self-reported emotional response during the adult attachment interview. American Psychological Association, 40, 776-789.

Sammer, G., Blecker, C., Gebhardt, H., Kirsch, P., Stark, R., \& VAITL, D. (2005). Acquisition of typical EEG waveforms during fMRI: SSVEP, LRP, and frontal theta. Neurolmage, 24, 1012-1024.

UGNELL, H., \& ÖBERG, P. A. (1995). The time-variable photoplethysmographic signal: Dependence of the heart synchronous signal on wavelength and sample volume. Medical Engineering \& Physics, 17, 571-578.

Van Audekerke, J., Peerters, R., Verhoye, M., Sijbers, J., \& Van DER LINDEN, A. (2000). Special designed RF-antenna with integrated non-invasive carbon electrodes for simultaneous magnetic resonance imaging and electro-encephalography acquisition at 7T. Magnetic Resonance Imaging, 18, 887-891.

von Smekal, A., Seelos, K. C., KÜper, C. R., \& Retser, M. (1995). Patient monitoring and safety during MRI examinations. European Radiology, 5, 302-305.

Williams, L. M., Phillifs, M. L., Brammer, M. J., Skerrett, D., LAgopoulos, J., ReNNIE, C. J., ET AL. (2001). Arousal dissociates amygdala and hippocampal fear responses: Evidence from simultaneous fMRI and skin conductance recording. Neurolmage, 14, 1070-1079.

Woodman, R. J., KINGWEll, B. A., BeIlin, L. J., Hamitron, S. E., DART, A. M., \& WATTS, G. F. (2005). Assessment of central and peripheral arterial stiffness. American Journal of Hypertension, 18, 249-260.

(Manuscript received February 2, 2007; revision accepted for publication May 10, 2007.) 